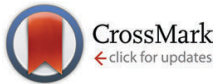

Cite this: Phys. Chem. Chem. Phys., $2015,17,6114$

Received 10th December 2014, Accepted 21st January 2015

DOI: $10.1039 / c 4 c p 05776 g$

www.rsc.org/pccp

\title{
Buckybowl superatom states: a unique route for electron transport?†
}

\author{
L. Zoppi, ${ }^{a}$ L. Martin-Samos ${ }^{\mathrm{bc}}$ and K. K. Baldridge ${ }^{{ }^{a}}$
}

A unique paradigm for intermolecular charge transport mediated by diffuse atomic-like orbital (SAMOs), typically present in conjugated hollow shaped molecules, is investigated for $\mathrm{C}_{20} \mathrm{H}_{10}$ molecular fragments by means of $G_{0} W_{0}$ theory. Inclusion of many body screening and polarization effects is seen to be important for accurate prediction of electronic properties involving these diffuse orbitals. Theoretical predictions are made for the series of bowl-shaped fullerene fragments, $\mathrm{C}_{20} \mathrm{H}_{10}, \mathrm{C}_{30} \mathrm{H}_{10}, \mathrm{C}_{40} \mathrm{H}_{10}, \mathrm{C}_{50} \mathrm{H}_{10}$. Interesting results are found for the LUMO-SAMO energy gap in $\mathrm{C}_{20} \mathrm{H}_{10}$, which is shown to be nearly an order of magnitude lower that that determined for $\mathrm{C}_{60}$. Given the ability to support bowl fragments on metal surfaces, these results suggest the concrete possibility for exploiting SAMO-mediated electron transport in supramolecular conducting layers.

\section{Introduction}

Renewed interest in the use of $\pi$-conjugated molecules as components in nanoscale electronics and optoelectronic devices $^{1}$ has motivated recent efforts to construct singlemolecule junctions that optimize transport properties. ${ }^{1 d, 2}$ Aromatic conjugated fragments are appealing for use in device technology due to their low density, structural stability, and extended-delocalized $\pi$ networks that support mobile charge carriers. ${ }^{3}$ As conductor dimensions approach the nanoscale, design principles focus towards producing molecules with tunable functionality ${ }^{4}$ to enable control of charge transport at the molecular scale. ${ }^{1 c, d}$ As such, the need has emerged for improved understanding of the details involved in optimization and control of electronic transport phenomena in these systems.

The extended family of curved aromatics based on the smallest bowl-shaped fullerene fragment, corannulene, $\mathrm{C}_{20} \mathrm{H}_{10}{ }^{3,5}$ (Fig. 1(a)), are key targets of interest. Much effort has been extended towards the design of functionalized building blocks based on this fragment, focusing on tailoring electronic properties such as electrical and optical band gaps. ${ }^{6}$ Assembled in the solid state, these bowl fragments provide an array of materials supported in varying complex environments, which can be exploited as active molecular layers in optoelectronic applications, ${ }^{7}$ aggregated as monolayers

\footnotetext{
${ }^{a}$ Department of Chemistry, University of Zurich, Winterthurerstrasse 190, Zurich, CH-8057, Switzerland. E-mail: kimb@oci.uzh.ch

${ }^{b}$ University of Nova Gorica, Materials Research Laboratory, vipavska cesta 11C, Ajdovscina 5270, Slovenia

${ }^{c}$ DEMOCRITOS, Istituto Officina dei Materiali, c/o SISSA Scuola Internazionale Superiore di Studi Avanzati, Via Bonomea 265, 34136 Trieste, Italy

$\dagger$ Electronic supplementary information (ESI) available. See DOI: 10.1039/ c4cp05776g
}

(a)

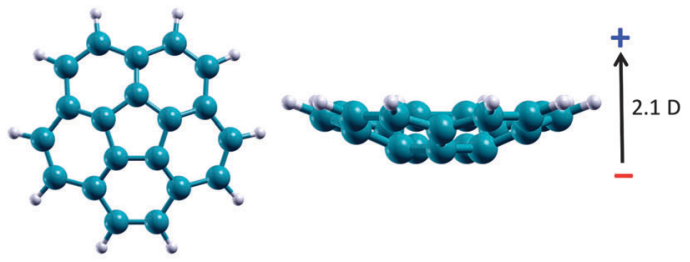

(b)

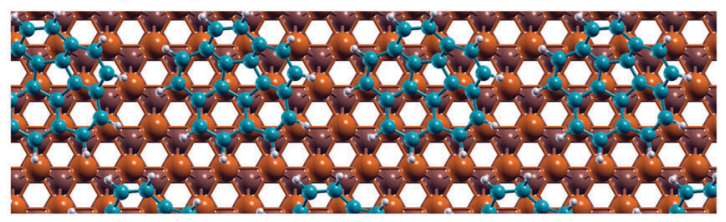

(c)

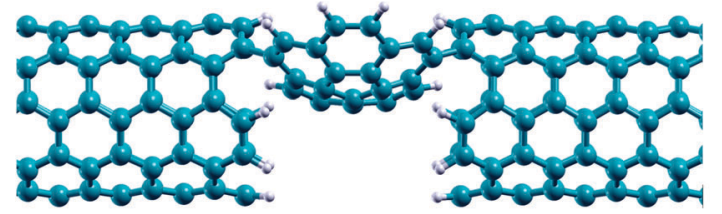

Fig. 1 (a) $\mathrm{C}_{20} \mathrm{H}_{10}$ molecular structure showing the shallow bowl and intrinsic dipole; (b) $\mathrm{C}_{20} \mathrm{H}_{10}$ molecules arranged on a $\mathrm{Cu}(111)$ surface; (c) $\mathrm{C}_{20} \mathrm{H}_{10}$ molecule assembled into a molecular junction.

on metallic surfaces for work-function engineering, ${ }^{8}$ or, as single molecules in junctions for transport processes (Fig. 1). ${ }^{9}$

Importantly, materials based on curved or hollow aromatic complexes enable exploitation of a unique mechanism of intermolecular charge transport distinct from the conventional mechanism involving tightly bound $\pi$ molecular orbital overlap. Key to these electron transport routes is evidence of a characteristic set of diffuse molecular orbitals called Super Atomic Molecular Orbitals, SAMOs, ${ }^{10}$ first investigated in the closed hollow aromatic molecule, $\mathrm{C}_{60} \cdot{ }^{10,11}$ SAMOs are virtual orbitals that arise 
from the central potential of the hollow molecular core, evoking well-defined hydrogen-like s, p, and d orbital angular momentum shapes. These orbitals extend well beyond the more tightly bound $\pi$ orbitals, and are also distinct from conventional Rydberg type orbitals that see the core as a point charge. ${ }^{11 b}$

The significance of SAMOs lies in the possibility for exploitation of the nearly free conducting channels, which arise when the molecular units are assembled in series in quantum nanostructures or solids. ${ }^{11 c}$ Such channels have been observed in low temperature scanning tunneling microscopy experiments (LT-STM) in the case of $\mathrm{C}_{60}$ molecules assembled on noble metal surfaces. ${ }^{10}$ Unfortunately, prospects for exploiting the SAMOs in practical applications must be tempered by the fact that typically these orbitals are unoccupied. In the case of $\mathrm{C}_{60}$, the SAMOs lie several $\mathrm{eV}$ above the lowest unoccupied molecular orbital, LUMO, and therefore are difficult to exploit for this purpose (Fig. 2). ${ }^{10}$

Taking a slightly different view from typically studied spheroid fullerenes, the present work details a theoretical investigation of the electronic properties of SAMOs in a series of curved shaped aromatic constructs, $\mathrm{C}_{20} \mathrm{H}_{10}, \mathrm{C}_{30} \mathrm{H}_{10}, \mathrm{C}_{40} \mathrm{H}_{10}, \mathrm{C}_{50} \mathrm{H}_{10}$, with focus on their suitability for applications in molecular circuits (electron transport). The intriguing electronic properties of the parent system, $\mathrm{C}_{20} \mathrm{H}_{10}$, can be ascribed to its large intrinsic dipole moment (2.01 D) and shallow bowl depth $(0.87 \AA),{ }^{12}$ which imparts dynamic properties characterized by a bowl-to-bowl inversion barrier of $11.5 \mathrm{kcal} \mathrm{mol}{ }^{-1} \cdot{ }^{12}$ The ability to control curvature (and therefore reactivity and properties) through functionalization of the rim, ${ }^{12}$ together with the possibility of assembling these building blocks in layers on metallic surfaces, ${ }^{8,13}$ strongly motivates their creative use as materials for supramolecular conducting layers.

\section{Results and discussion}

\section{SAMOs: theoretical description}

The physical origin of SAMOs is ascribed to many-body screening and polarization effects, typical of a polarizable assembly (e.g., graphene) that undergoes a topological distortion, such as wrapping or rolling into a nanotube or fullerene. ${ }^{14}$ At a solidvacuum interface, these many body interactions give rise to a series of degenerate image potential (IP) states in the nearsurface region on both sides of a graphene sheet, which float above and below the molecular plane and undergo free motion parallel to it. ${ }^{14}$ Topological distortion of the molecular sheet

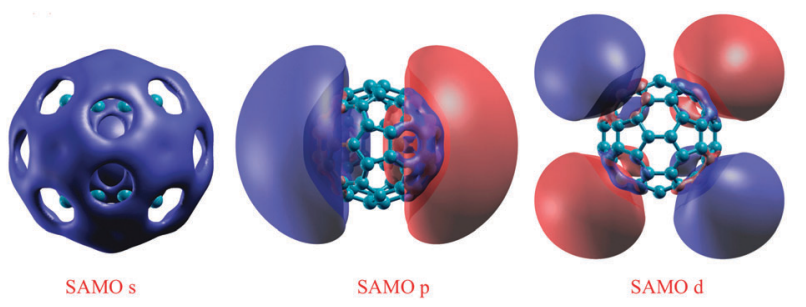

Fig. 2 DFT-LDA calculated SAMOs, illustrating the typical hydrogen-like $\mathrm{s}, \mathrm{p}$, and $\mathrm{d}$ symmetric shapes in $\mathrm{C}_{60}$. breaks symmetry, lowering/raising the energy of the IP states on the concave/convex side of the resulting material, revealing SAMOs. $^{11 b}$

Theoretical investigation of SAMOs requires methods that properly include polarization and correlation effects, which are crucial for describing image-potential states and are typically poorly described with conventional density functional theory (DFT) approaches ${ }^{15}$ due to the approximate nature of the exchange and correlation (XC) potential. ${ }^{16}$ In order to capture these effects, the choice of the methodology falls to many body perturbation theory (MBPT) ${ }^{17}$ electronic structure approaches within the $G W$ scheme. ${ }^{18}$ In this approximation, ${ }^{17,18}$ the selfenergy, $\Sigma$, is the product of a single-particle Green function, $G$, and a nonlocal and dynamically screened Coulomb potential, $W,(\Sigma=i G W)$. Due to the high computational demands of fully selfconsistent $G W(\operatorname{sc} G W)$, a range of perturbative $G W$ schemes, from non self-consistent to partially self-consistent have emerged. ${ }^{19}$ The lowest rung in this hierarchy, standard in practical calculations, is a non-self-consistent scheme, i.e. $G_{0} W_{0},{ }^{19,20}$ where the quasi particle (QP) energies are obtained as a perturbative first-order correction to the DFT eigenvalues. It is important to note that $G W$ theory is an approximation for $G W \Gamma$ theory (former work of Hedin and Lunquist). ${ }^{18}$ It is not straightforward that full $\operatorname{sc} G W$ could provide better QP energies than any perturbative $G W$ scheme.

The use and appropriateness of the $G W$ method for prediction of image potential states has raised some skepticism in the QM community. However, as well documented in the literature, we also strongly believe that the $G W$ methodology is suitable to describe many-body screening and long-range polarization effects crucial for treating image-potential states. ${ }^{21}$ A further criticism can derive from the use of DFT wavefunctions for the purpose of SAMO description. However, differences between the Kohn and Sham and real QP wavefunction come from the incorrect longrange behavior of approximate exchange potentials in conventional DFT schemes, which scarcely effects the $G W$-corrected QP energies. $^{22}$ In this respect, it is worth noting that the first theoretical investigation of SAMOs, successfully compared to experiments, was performed within a DFT based scheme. ${ }^{10}$

Unfortunately, the non-self-consistency in $G_{0} W_{0}$ can give rise to a dependence of the resulting QP spectra from the starting DFT functional, as recently documented in investigations of QP valence spectra of molecules. ${ }^{19,23}$ Recent efforts are being directed at the performance of $G_{0} W_{0}$ schemes across specific benchmark sets of molecules used in organic electronic devices. ${ }^{19,23 a}$ Although still controversial, emerging from these investigations is the strategy of including a fraction of exact-exchange (EXX) in hybrid-functionals to mitigate the self-interaction error (SIE), thereby providing an improved starting point for $G_{0} W_{0}$ calculation. ${ }^{19,23 c}$ For example, a recent investigation involving azabenzenes showed good agreement with photoemission experiments with this strategy. ${ }^{19,23 c}$ Such studies are still in their infancy, however, with available data primarily involving QP valence spectra, whereas predictions involving the virtual unoccupied space still motivates further investigation.

A second controversial point concerning appropriate methodology for investigation of SAMOs derives from the fact that 
some QM-based methods (e.g., equation of motion (EOM) methodology ${ }^{24}$ ) include higher order correlation than $G_{0} W_{0}$. However, such approaches are based on series expansions in terms of the bare Coulomb potential and therefore require higher order in the diagrammatic expansion to be able to capture the correlation effects. The $G W$ methodology, on the other hand, is based on a series expansion in terms of a screened Coulomb potential, with the polarizability calculated within RPA and the screening through the inversion of a Dyson equation, which by definition, includes an infinite number of diagrams. ${ }^{18 a}$ As such, results coming from QM methods are quite sensitive to the order in the diagrammatic expansion, whereas such sensitivity does not appear in the $G W$ approach. Moreover, the $G W$ method is highly predictive with less computational effort even for systems with high dielectric constants.

In the present work, a customized hybrid methodology is exploited, using standard B97D/Def2-TZVPP ${ }^{25}$ (GAMESS) ${ }^{26}$ DFT for full optimization and Hessian characterization of structures, followed by plane-wave DFT formalism (Quantum-ESPRESSO) ${ }^{27}$ within $\mathrm{MBPT}^{17}$ in the $G W$ approximation ${ }^{18}$ (SAX). ${ }^{28}$ This method is applied to a series of $\mathrm{C}_{20} \mathrm{H}_{10}$ based systems to investigate and compare SAMOs and SAMO electronic properties. In addition, the performance dependence of the $G_{0} W_{0}$ scheme on the starting functional for the $G W$ calculation is compared using both a DFT-PZ functional ${ }^{29}\left(G_{0} W_{0} @ L D A\right)$ as well as a hybrid PBE0 functional $^{30}\left(G_{0} W_{0} @\right.$ PBE0) that includes $25 \%$ exact exchange (EXX) (for additional details see ESI $\dagger$ ).

\section{SAMOs in $\mathrm{C}_{20} \mathrm{H}_{10}$}

Calculated SAMOs of corannulene are depicted in Fig. 3. As has been shown for the closed shaped hollow $\mathrm{C}_{60}$ structure, corannulene also manifests the characteristic diffuse molecule-centered hydrogenic-like s, p, and d shapes. To be relevant for charge transport, the SAMO derived states should cross the Fermi level in any resulting material. To facilitate this characteristic, the lowest energy SAMO of the isolated molecule should lie close to the LUMO. Thus, the ability to control the LUMO and SAMOs gap of lowest energy in a molecular system becomes quite important.

$G_{0} W_{0}$ as well as DFT predictions of $\Delta E_{\text {SAMO-LUMO }}$ for $\mathrm{C}_{20} \mathrm{H}_{10}$ are summarized in Table 1 . As in $\mathrm{C}_{60}$, SAMOs are revealed in the unoccupied part of the spectrum in a simple DFT calculation (LDA or PBE0). Inclusion of many-body effects at the $G_{0} W_{0}$ level provides a more accurate positioning of SAMO levels with respect to conventional DFT predictions, but still is dependent
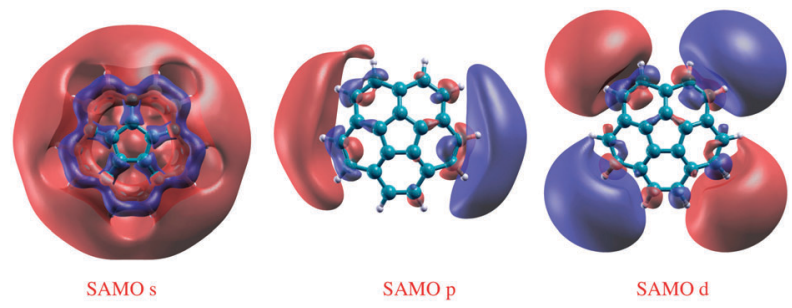

Fig. 3 DFT-LDA 3D representation of SAMOs illustrating the typical s, p, and d-like symmetric shapes in corannulene, $\mathrm{C}_{20} \mathrm{H}_{10}$.
Table 1 Comparison of $\Delta E_{\text {SAMO-LUMO }}$ predictions for $\mathrm{C}_{20} \mathrm{H}_{10}$ at different levels of theory, in eV

\begin{tabular}{lllll}
\hline & \multicolumn{4}{l}{$\Delta E_{\text {SAMO-LUMO }}(\mathrm{eV})$} \\
\cline { 2 - 5 } SAMO & DFT(LDA $)$ & DFT(PBE0) & $G_{0} W_{0} @ L D A$ & $G_{0} W_{0} @$ PBE0 \\
\hline $\mathrm{s}$ & 2.3 & 1.8 & 0.3 & -0.7 \\
$\mathrm{p}$ & 2.5 & 2.0 & 0.4 & -0.6 \\
$\mathrm{~d}$ & 2.8 & 2.3 & 0.5 & -0.3
\end{tabular}

on the starting functional. Initialization of the $G W$ calculation from a standard DFT-PZ functional ( $\left.G_{0} W_{0} @ L D A\right)$ results in SAMO level of s-type symmetry that corresponds to the 1st unoccupied level after the LUMO. On the other hand, initialization of the $G W$ calculation from a hybrid PBE0 functional that includes a percentage of exact exchange, $\left(G_{0} W_{0} @ P B E 0\right)$, predicts all SAMO orbitals to be lower in energy than the LUMO. The origin of the starting point dependence in $G_{0} W_{0}$ can be traced back to differences in the orbitals and orbital energies used as input for the self-energy calculation. In particular, the Coulomb potential, $W$, being roughly inversely proportional to the occupied $\rightarrow$ unoccupied transition energies, is extremely sensitive to any over-(under-)estimation of the HOMO-LUMO gap, which generally results in an under-(over-)estimation of screening.

Notably, the $\Delta E_{\text {SAMO-LUMO }}$ energy gap for SAMO orbital of s-type symmetry in $\mathrm{C}_{20} \mathrm{H}_{10}$ is predicted to be $\sim 0.3 \mathrm{eV}$, nearly an order of magnitude smaller than in $\mathrm{C}_{60}$ (calc. $2.4 \mathrm{eV}$ ). ${ }^{11 c}$ This proximity of the LUMO with respect to the delocalized SAMOs in $\mathrm{C}_{20} \mathrm{H}_{10}$, suggests possibilities for exploiting SAMO-mediated electron transport in a material using these bowl fragments as functional units.

Unfortunately, there are as of yet no reported experimental data concerning the electronic properties of SAMOs for the case of $\mathrm{C}_{20} \mathrm{H}_{10}$. However, one can make relevant comparisons of HOMO-LUMO gap given available ionization potential (IP) and electron affinity (EA) measurements that have been made experimentally. ${ }^{31}$ Depending on the experimental technique used, variations in HOMO-LUMO gap (IP-EA) range from 7.6 to $8.0 \mathrm{eV} .^{31}$ The corresponding calculated values for the HOMO-(molecular)LUMO gap, considering the different DFT starting points, range from $7.3 \mathrm{eV}\left(G_{0} W_{0} @ \mathrm{LDA}\right)$ to $8.1 \mathrm{eV}$ $\left(G_{0} W_{0} @\right.$ PBE0). This provides a meaningful comparison with experiment, and establishes the level of accuracy of the described customized hybrid methodology.

\section{Doping effects on SAMOs levels}

The optimal size and shape of the small aromatic fragment, $\mathrm{C}_{20} \mathrm{H}_{10}$, provides characteristic features complementary, but unique, to that of the fullerenes or graphene structures. In particular, the LUMO-SAMO gap shows an important decrease with respect to $\mathrm{C}_{60}\left(0.3 \mathrm{eV} v s .2 .4 \mathrm{eV}\right.$ in $\left.\mathrm{C}_{60}\right) .{ }^{11 c}$

Consideration of SAMOs for electron transport does not necessarily require one to focus on ' $a d$ hoc' charged systems, as are sometimes considered. ${ }^{32}$ We instead propose a strategy for doping $\mathrm{C}_{20} \mathrm{H}_{10}$ in order to occupy the molecular LUMOs, for the purpose of enhancing the SAMO occupation. In fact, a 
further reduction of the LUMO-SAMO gap can be achieved via a modification of the central hollow potential of the molecular cage, which ultimately defines the SAMO wavefunctions. ${ }^{11 a}$ In this respect, internal (endohedral) doping with electron donating metal atoms has been suggested as a means to substantially reduce the LUMO-SAMO gap when the ionization potential of the endohedral atom is sufficiently large, as recently shown for $\mathrm{C}_{60} \cdot{ }^{11 a}$ Interestingly, external (exohedral) doping is quite ineffective in reducing the SAMO energy, because the molecular cavity actually acts as a Faraday cage, effectively screening the states that are confined by the cage from the presence of any external charge. ${ }^{11 a}$

$\mathrm{C}_{20} \mathrm{H}_{10}$ has been previously shown to have strong electronacceptor character and forms stable complexes with alkaline metals, accommodating up to four electrons in the doubly degenerate LUMOs. ${ }^{13 b}$ Photoelectron spectra together with computations have shown $\mathrm{C}_{20} \mathrm{H}_{10}$ complexes doped with Cs and deposited on a $\mathrm{Cu}(111)$ surface to have full occupation of the original $\mathrm{C}_{20} \mathrm{H}_{10}$ degenerate LUMOs. ${ }^{13 b}$ Since cage-doping with metals having large ionization potentials (e.g., $\mathrm{Li}, \mathrm{Na}$ ) are suggested as the most effective for tuning the SAMO-LUMO energy gap, ${ }^{11 a}$ it is of interest to investigate the corresponding effects in $\mathrm{C}_{20} \mathrm{H}_{10}$ complexes with these particular metal atoms.

Three Li-doped $\mathrm{C}_{20} \mathrm{H}_{10}$ complexes are considered in this work, differing in the way the $\mathrm{Li}$ atoms are complexed to the molecular cage: (1) two Li atoms complexed to the convex side and two on the concave side; (2) all $4 \mathrm{Li}$ atoms complexed to the convex side (exohedral); and (3) all $4 \mathrm{Li}$ atoms complexed to the concave side (endohedral). The B97D//Def2-TZVPP fully optimized gas phase structures are shown in Fig. 4.

First, it is of interest to compare the basic molecular orbital structure of the un-doped $\mathrm{C}_{20} \mathrm{H}_{10}$ with that of the $4 \mathrm{Li}$-doped complexes. Due to the decrease in symmetry, the two degenerate LUMOs in the un-doped molecule are no longer degenerate in all Li-doped complex. In the doped Li-complexes, these orbitals are now occupied (Li-doped $\mathrm{C}_{20} \mathrm{H}_{10}, 2 \mathrm{Li}$ convex, $2 \mathrm{Li}$ concave and $\mathrm{C}_{20} \mathrm{H}_{10}, 4 \mathrm{Li}$ concave) as $\mathrm{HOMO}-1$ and HOMO, or frontier molecular orbitals (Li-doped $\mathrm{C}_{20} \mathrm{H}_{10}, 4 \mathrm{Li}$ convex). Fig. 5 illustrates the two degenerate LUMOs in the parent molecule
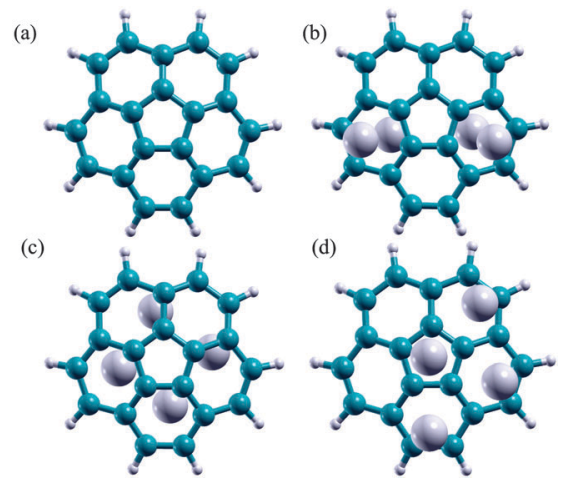

Fig. 4 (a) Un-doped $\mathrm{C}_{20} \mathrm{H}_{10}$, (b) Li-doped $\mathrm{C}_{20} \mathrm{H}_{10}, 2 \mathrm{Li}$ convex side, $2 \mathrm{Li}$ concave side, (c) Li-doped $\mathrm{C}_{20} \mathrm{H}_{10} 4 \mathrm{Li}$ concave side, (d) Li-doped $\mathrm{C}_{20} \mathrm{H}_{10} 4$ Li convex side.

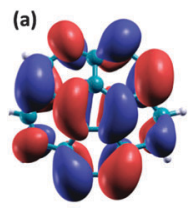

(b)

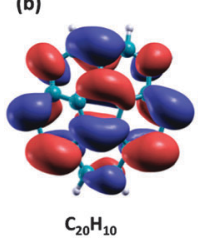

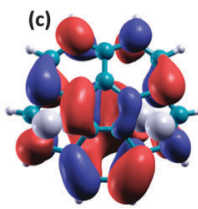

(d)

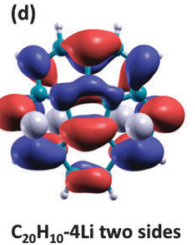

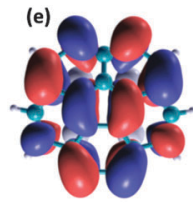

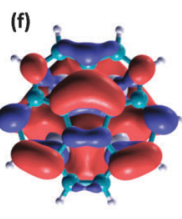

$\mathrm{C}_{20} \mathrm{H}_{10}-4 \mathrm{Li}$ (concave)
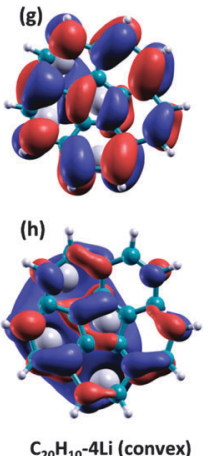

Fig. 5 (a) and (b) degenerate LUMOs in $\mathrm{C}_{20} \mathrm{H}_{10}$, (c) and (d) $\mathrm{HOMO}$ and $\mathrm{HOMO}-1$ in $\mathrm{Li}$-doped $\mathrm{C}_{20} \mathrm{H}_{10}, 2 \mathrm{Li}$ convex side and $2 \mathrm{Li}$ on concave side, (e) and (f) $\mathrm{HOMO}$ and $\mathrm{HOMO}-1$ in $\mathrm{Li}$-doped $\mathrm{C}_{20} \mathrm{H}_{10} 4 \mathrm{Li}$ on concave side, (e) and (f) $\mathrm{HOMO}$ and $\mathrm{HOMO}-1$ in $\mathrm{Li}$-doped $\mathrm{C}_{20} \mathrm{H}_{10} 4 \mathrm{Li}$ on convex side.

compared to the now-occupied orbitals in the 3 Li-doped complexes.

The occupation of the pristine molecular LUMOs in the $\mathrm{Li}$ complexes has a dramatic effect in closing the HOMO-LUMO gap with respect to that of the un-doped corannulene. The 'new HOMOs' in the hybrid systems are higher in energy, and, considering the GW@LDA HOMO-LUMO gap of $\mathrm{C}_{20} \mathrm{H}_{10}(7.3 \mathrm{eV})$ versus the corresponding value of the Li-doped structures (Table 2), one sees a decrease on the order of a factor of 2 .

A second point of interest is to address the effect of $\mathrm{Li}$ doping on the SAMO-LUMO energy gap. In particular, it is of interest to investigate the Li-doped $\mathrm{C}_{20} \mathrm{H}_{10}, 4 \mathrm{Li}$ concave structure, which due to the endohedral doping should be the most effective for tuning purposes in this respect. Moreover, the atomic arrangement of the $\mathrm{Li}$ atoms on the concave side of the molecular cage is symmetric and, as such, less perturbing to the SAMOs of $\mathrm{p}$ and $\mathrm{d}$ symmetry, which extend outside the central molecular core and are easily recognizable.

The calculated $\Delta E_{\text {SAMO-Lumo }}$ data is reported in Table 3. According to these results, the Li doping is seen not to decrease the SAMO-LUMO gap compared to the parent molecule at the $G_{0} W_{0}$ level, independent of starting functional. In fact, considering the $G_{0} W_{0} @ L D A\left(G_{0} W_{0} @ P B E 0\right)$ SAMOs-LUMO gap of $\mathrm{C}_{20} \mathrm{H}_{10}$ (Table 1) versus the corresponding values of the Li-doped structures (Table 3), one sees that $\mathrm{C}_{20} \mathrm{H}_{10} \Delta E_{\text {SAMO-Lumo values are }}$ lower than those of the Li-doped complex by $0.5 \mathrm{eV}(0.8 \mathrm{eV})$ for $\mathrm{p}$ symmetry SAMOs and by $0.7 \mathrm{eV}(0.9 \mathrm{eV})$ for d symmetry SAMOs.

The above analysis shows again the strong electron-acceptor character of $\mathrm{C}_{20} \mathrm{H}_{10}$, which accommodates 4 electrons in the double-degenerate LUMOs when complexed with Li. Other than

Table 2 HOMO-LUMO gap predictions for the Li-doped $\mathrm{C}_{20} \mathrm{H}_{10}$ complexes at different level of theory

\begin{tabular}{llll}
\hline & \multicolumn{4}{l}{ HOMO-LUMO GAP $(\mathrm{eV})$} \\
\cline { 2 - 4 } Structure & DFT-LDA & PBE0 & $G_{0} W_{0} @ L D A$ \\
\hline $\mathrm{C}_{20} \mathrm{H}_{10}$-4Li-convex & 0.2 & 1.2 & 3.7 \\
$\mathrm{C}_{20} \mathrm{H}_{10}$-4Li-concave & 0.4 & 1.5 & 3.4 \\
$\mathrm{C}_{20} \mathrm{H}_{10}$-4Li-2sides & 0.4 & 1.3 & 3.0
\end{tabular}


Table $3 \Delta E_{\text {SAMO-LUMO }}$ predictions for the Li-doped $\mathrm{C}_{20} \mathrm{H}_{10} 4 \mathrm{Li}$ concave complex at different levels of theory

\begin{tabular}{lllll}
\hline \multirow{2}{*}{ SAMO } & \multicolumn{4}{l}{$\Delta E_{\text {SAMO-LUMO }}(\mathrm{eV})$} \\
\cline { 2 - 5 } & $\mathrm{DFT}(\mathrm{LDA})$ & $\mathrm{DFT}(\mathrm{PBE})$ & $G_{0} W_{0} @ \mathrm{LDA}$ & $G_{0} W_{0} @$ PBE0 \\
\hline $\mathrm{p}$ & 2.5 & 2.1 & 0.9 & 0.2 \\
$\mathrm{~d}$ & 2.7 & 2.0 & 1.2 & 0.6
\end{tabular}

the dramatic decrease in HOMO-LUMO gap and loss of the p, d quasi degeneracy, doping with Li does not significantly affect the LUMO-SAMOs gap.

\section{SAMOs in fullerene fragments}

Consideration of electronic and transport properties across the full series of molecules of increased curvature, $\mathrm{C}_{20} \mathrm{H}_{10}-\mathrm{C}_{50} \mathrm{H}_{10}$, becomes of interest based on the results for the smallest of the series, $\mathrm{C}_{20} \mathrm{H}_{10}$, shown above. The presence of strong intrinsic molecular dipoles manifested by the curvature in these molecular fragments, together with the increasingly large polarizable surface of $\pi$ electron density, are fundamental to unlocking and exploiting such systems for material devices. ${ }^{9}$ The increasing bowl depth and change in curvature across the series shows systematic trends in structure and property towards a tube structure (Fig. 6). ${ }^{9}$ It is of interest, therefore, to see if the same trends can be observed in SAMOs related electronic properties.

As shown in previous work for shallow molecular bowls, ${ }^{9}$ dipoles induced across the relatively large surface area of the cap (pentagon) region may become comparable or larger than the intrinsic molecular dipole (perpendicular to the cap). On the other hand, for deeper bowls such an effect is not so apparent, reflecting the increased conjugated area of the belt region while approaching what one might find in a tube-like structure. To fully understand how the structural and electronic transition between the bowl-like structure $\left(\mathrm{C}_{20} \mathrm{H}_{10}\right)$ to the tubelike $\left(\mathrm{C}_{50} \mathrm{H}_{10}\right)$ structure can possibly affect the SAMO electronic properties, $G_{0} W_{0}$ calculations have been undertaken for investigating SAMOs across the series $\mathrm{C}_{30} \mathrm{H}_{10}-\mathrm{C}_{50} \mathrm{H}_{10}$. The $G_{0} W_{0} @ L D A$ predicted SAMOs-LUMO gap energy values for the series are summarized in Table 4 and Fig. 7. The corresponding orbital depictions are illustrated in Fig. 8.

Comparison of the SAMO-LUMO energy gaps shows a sharp increase from $\mathrm{C}_{20} \mathrm{H}_{10}$ to $\mathrm{C}_{30} \mathrm{H}_{10}$, followed by a more modest

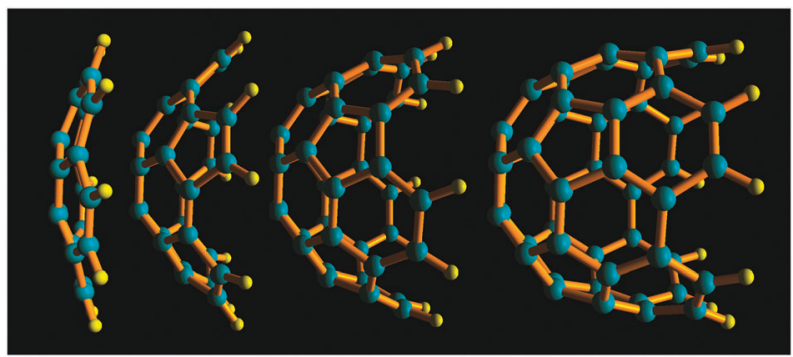

Fig. 6 Curved aromatic bowl constructs of increasing size and depth, based on the smallest corannulene unit (left most).
Table $4 \Delta E_{\text {SAMO-LUMO }}$ predictions for $\mathrm{C}_{20} \mathrm{H}_{10}, \mathrm{C}_{30} \mathrm{H}_{10}, \mathrm{C}_{40} \mathrm{H}_{10}, \mathrm{C}_{50} \mathrm{H}_{10}$ at the $G_{0} W_{0} @$ aLDA level of theory, in eV

\begin{tabular}{lll}
\hline System & SAMO type & $\Delta E_{\text {SAMO-LUMO }}$ \\
\hline $\mathrm{C}_{20} \mathrm{H}_{10}$ & $\mathrm{~s}$ & 0.3 \\
& $\mathrm{p}$ & 0.4 \\
& $\mathrm{~d}$ & 0.5 \\
$\mathrm{C}_{30} \mathrm{H}_{10}$ & & \\
& $\mathrm{~s}$ & 1.3 \\
& $\mathrm{p}$ & 1.9 \\
$\mathrm{C}_{40} \mathrm{H}_{10}$ & $\mathrm{~d}$ & 1.7 \\
& & \\
& $\mathrm{~s}$ & 2.0 \\
& $\mathrm{p}$ & 2.2 \\
$\mathrm{C}_{50} \mathrm{H}_{10}$ & $\mathrm{~d}$ & 2.6 \\
& & \\
& $\mathrm{~s}$ & 1.6 \\
& $\mathrm{p}$ & 2.1 \\
& $\mathrm{~d}$ & 2.5 \\
\hline
\end{tabular}

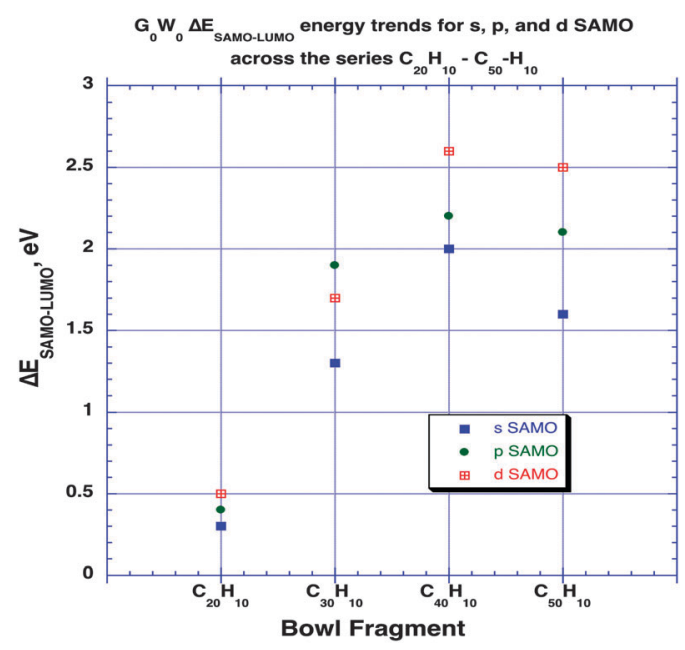

Fig. 7 Trends in $G_{0} W_{0} @ L D A$ predicted $\Delta E_{\text {SAMO-LUMO }}$ across the series of aromatic bowl constructs of increasing size and depth, $\mathrm{C}_{20} \mathrm{H}_{10}, \mathrm{C}_{30} \mathrm{H}_{10}$, $\mathrm{C}_{40} \mathrm{H}_{10}, \mathrm{C}_{50} \mathrm{H}_{10}$, in eV.

increase to $\mathrm{C}_{40} \mathrm{H}_{10}$, and then a significant drop for the more tube-like structure, $\mathrm{C}_{50} \mathrm{H}_{10}$ (e.g., Fig. 7). For the lowest energy s-type SAMO, the gap relative to $\mathrm{C}_{20} \mathrm{H}_{10}(0.3 \mathrm{eV})$ increases by $1.0 \mathrm{eV}$ for $\mathrm{C}_{30} \mathrm{H}_{10}$, by $1.7 \mathrm{eV}$ for $\mathrm{C}_{40} \mathrm{H}_{10}$, and then levels off to an increase of $1.3 \mathrm{eV}$ for $\mathrm{C}_{50} \mathrm{H}_{10}$. Importantly, in the case of the smallest bowl, $\mathrm{C}_{20} \mathrm{H}_{10}$, this energy gap is still one order of magnitude lower than the next higher analogue, $\mathrm{C}_{30} \mathrm{H}_{10}$. SAMOs of $\mathrm{p}$ and d-type symmetry are significantly higher in energy than the LUMO (from $1.7 \mathrm{eV}$ up to $2.6 \mathrm{eV}$ ), and do not show promise for enhancing the occupation of the delocalized orbitals.

Finally, one can observe where $\mathrm{C}_{60}$ fits in the series in terms of 'curvature' and resulting $\Delta E_{\mathrm{SAMO}}$-LUMO with respect to the series, as shown in Fig. 9. One finds that the value of $\Delta E_{\text {SAMO-LUMO }}$ at $2.4 \mathrm{eV}$ is still on the dramatic increase in trend of the bowl structures shown in Fig. 7, before the falloff towards the tube construct of $\mathrm{C}_{50} \mathrm{H}_{10}$. The local curvature of $\mathrm{C}_{60}$ also places it between $\mathrm{C}_{40} \mathrm{H}_{10}$ and $\mathrm{C}_{50} \mathrm{H}_{10}$, but closer to the latter. 


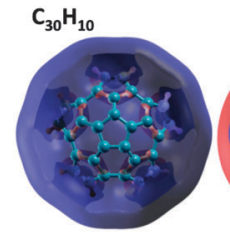

$\mathrm{C}_{40} \mathrm{H}_{10}$
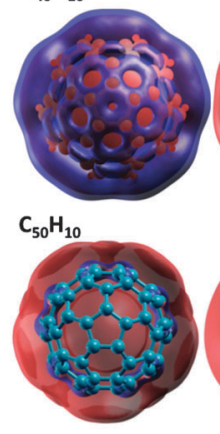

SAMO $s$ (a)

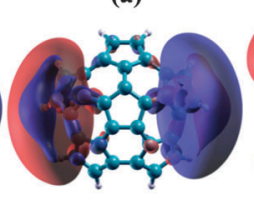

(b)

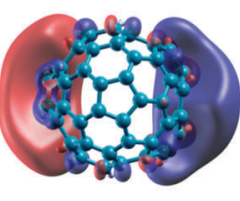

(c)

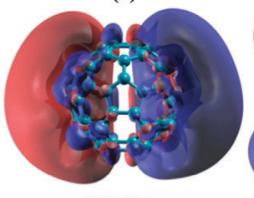

SAMO p
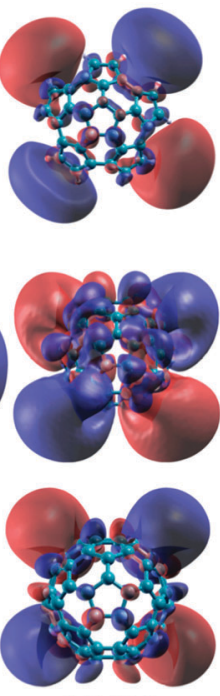

SAMO d
Fig. 8 DFT-LDA representation of SAMOs illustrating the typical $s, p$, d-like symmetric shapes in (a) $\mathrm{C}_{30} \mathrm{H}_{10}$, (b) $\mathrm{C}_{40} \mathrm{H}_{10}$, (c) $\mathrm{C}_{50} \mathrm{H}_{10}$.

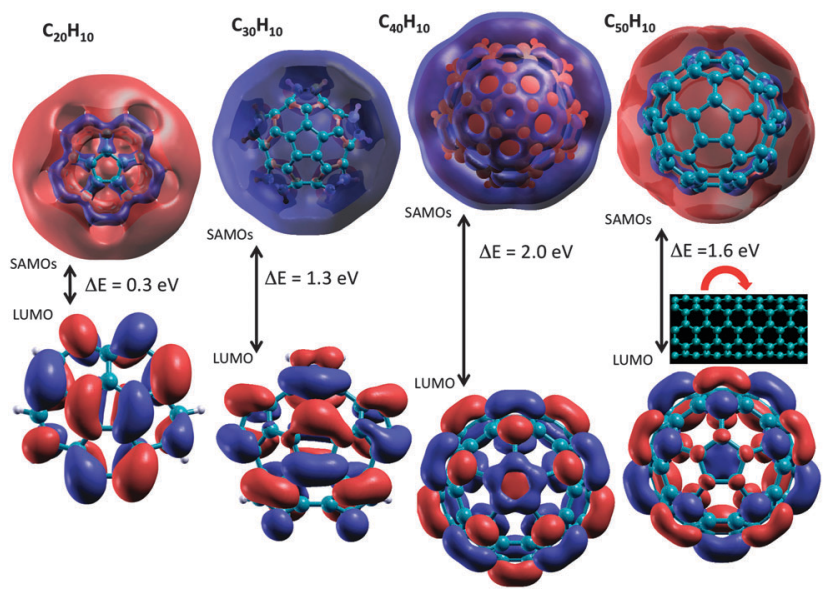

Fig. $9 G_{0} W_{0} @ L D A$ predicted trends across the series of buckybowls, $\mathrm{C}_{20} \mathrm{H}_{10}, \mathrm{C}_{30} \mathrm{H}_{10}, \mathrm{C}_{40} \mathrm{H}_{10}, \mathrm{C}_{50} \mathrm{H}_{10}$.

\section{Conclusions}

SAMO-type orbitals, shown to exist in conjugated hollowshaped molecule, offer an important opportunity to exploit new mechanisms for intermolecular electron transport. Taking a slightly different view from the typically studied $\mathrm{C}_{60}$, the present investigation reports theoretical evidence of the electronic properties of SAMOs in a series of bowl shape aromatic constructs, $\mathrm{C}_{20} \mathrm{H}_{10}-\mathrm{C}_{50} \mathrm{H}_{10}$, with focus on the suitability of such orbitals in electron transport applications. SAMO electronic properties are revealed using accurate many body screening and polarization effects at the $G_{0} W_{0}$ level, with consideration of the dependence of calculated results on the DFT starting functional.

$G_{0} W_{0}$ predictions of SAMO levels in $\mathrm{C}_{20} \mathrm{H}_{10}$ reveal a LUMOSAMO energy gap nearly an order of magnitude lower that that found in $\mathrm{C}_{60} \cdot{ }^{11 c}$ This finding is extremely important in supporting design of $\mathrm{C}_{20} \mathrm{H}_{10}$-based materials to enhance occupation of these diffuse orbitals in experimental investigations. Contrary to literature proposals for $\mathrm{C}_{60},{ }^{33} \mathrm{Li}$ doping in $\mathrm{C}_{20} \mathrm{H}_{10}$ does not reduce the LUMO-SAMO gap over that of the un-doped corannulene. However, the promising results for un-doped corannulene, as well as other prospects for doping in these constructs, warrants further investigation towards this direction.

Further analysis of SAMOs in higher order analogues of $\mathrm{C}_{20} \mathrm{H}_{10}$, revealed a sharply increasing trend in $\Delta E_{\text {SAMO-LUMO }}$ energy gap up to $\mathrm{C}_{40} \mathrm{H}_{10}$, followed by a levelling off when the bowl structure reaches the morphology of tube structure (e.g., $\mathrm{C}_{50} \mathrm{H}_{10}$ ). Results of the present work have motivated experimental efforts to look into SAMO structure of $\mathrm{C}_{20} \mathrm{H}_{10}$. However, investigation of $\mathrm{C}_{20} \mathrm{H}_{10}$ assembled on metallic surface with established LT-STM methods is nontrivial, and considerable efforts are still necessary to determine the appropriate surface type and associated experimental protocols to achieve the necessary resolution.

Studies such as the present investigation provide an important opportunity to establish strengths and limitations of customized and enhanced hybrid methodologies for accurate predictions of materials phenomenon. Given the scant literature on performance of theoretical strategies for prediction of transport phenomenon, it is desirable to continue efforts in this direction, as established theoretical approaches hold an important role in the improved understanding of fundamental mechanisms.

\section{Acknowledgements}

L.Z. and K.K.B. acknowledge the University of Zürich, the Swiss National Science Foundation, and the UZH-UFSP for support of this research. L. M.-S. acknowledges Prof. Erio Tosatti for useful discussions. CSCS supercomputing center is gratefully acknowledged for a grant of computer time.

\section{References}

1 (a) H. Uoyama, K. Goushi, K. Shizu, H. Nomura and C. Adachi, Highly efficient organic light-emitting diodes from delayed fluorescence, Nature, 2012, 492, 234-240; (b) D. 1. N. Congreve, J. Lee, N. J. Thompson, E. Hontz, S. R. Yost, P. D. Reusswig, M. E. Bahlke, S. Reineke, T. V. Voorhis and M. A. Baldo, External Quantum Efficiency Above 100\% in a Singlet-Exciton-Fission-Based Organic Photovoltaic Cell, Science, 2013, 340, 334-337; (c) H. Song, M. A. Reed and T. Lee, Single Molecule Electronic Devices, Adv. Mater., 2011, 23, 1583-1608; (d) S. V. Aradhya and L. Venkataraman, Single-molecule junctions beyond electronic transport, Nat. Nanotechnol., 2013, 8, 399-410.

2 (a) H. Vazquez, R. Skouta, S. Schneebeli, M. Kamenetska, R. Breslow, L. Venkataraman and M. S. Hybertsen, Probing the conductance superposition law in single-molecule circuits with parallel paths, Nat. Nanotechnol., 2012, 7, 663-667; 
(b) C. M. Guédon, H. Valkenier, T. Markussen, K. S. Thygesen, J. C. Hummelen and S. J. v. d. Molen, Observation of quantum interference in molecular charge transport, Nat. Nanotechnol., 2012, 7, 305-309.

3 L. Zoppi, J. S. Siegel and K. K. Baldridge, Electron transport and optical properties of curved aromatics, WIREs Comput. Mol. Sci., 2013, 3, 1-12.

4 (a) D. Chaudhuri, H. Wettach, K. J. V. Schooten, S. Liu, E. Sigmund, S. Höger and J. M. Lupton, Tuning the SingletTriplet Gap in Metal-Free Phosphorescent p-Conjugated Polymers, Angew. Chem., Int. Ed., 2010, 49, 7714-7717; (b) M. D. Valle, R. Gutiérez, C. Tejedor and G. Cuniberti, Tuning the conductance of a molecular switch, Nat. Nanotechnol., 2007, 2, 176-179; (c) G. Valenti, C. Bruno, S. Rapino, A. Fiorani, E. A. Jackson, L. T. Scott, F. Paolucci and M. Marcaccio, Intense and Tunable Electrochemiluminescence of Corannulene, J. Phys. Chem. C, 2010, 114, 19467-19472.

5 L. T. Scott, H. E. Bronstein, D. V. Preda, R. B. M. Ansems, M. S. Bratcher and S. Hagen, Geodesic polyarenes with exposed concave surfaces, Pure Appl. Chem., 1999, 71, 209-219.

6 Y.-T. Wu, D. Bandera, R. Maag, A. Linden, K. K. Baldridge and J. S. Siegel, Multi-ethynyl Corannulenes: Synthesis, Structure and Properties, J. Am. Chem. Soc., 2008, 130, 10729-10739.

7 (a) J. Mack, P. Vogel, D. Jones, N. Kaval and A. Sutton, The development of corannulene-based blue emitters, Org. Biomol. Chem., 2007, 5, 2448-2452; (b) L. Zoppi, L. MartinSamos and K. K. Baldridge, Effect of Molecular Packing on Corannulene-Based Materials Electroluminescence, J. Am. Chem. Soc., 2011, 133, 14002-14009.

8 T. Bauert, L. Zoppi, G. Koller, A. Garcia, K. K. Baldridge and K.-H. Ernst, Large interface dipole moments without charge transfer: buckybowls on metal surfaces, J. Phys. Chem. Lett., 2011, 2, 2805-2809.

9 L. Zoppi, A. Ferretti and K. K. Baldridge, Static and FieldOriented Properties of Bowl-Shaped Polynuclear Aromatic Hydrocarbon Fragments, J. Chem. Theory Comput., 2013, 9, 4797-4804.

10 M. Feng, J. Zhao and H. Petek, Atomlike, hollow-core-bound molecular orbitals of C60, Science, 2008, 320, 359-362.

11 (a) J. Zhao, M. Feng, J. L. Yang and H. Petek, The Superatom States of Fullerenes and Their Hybridization into the Nearly Free Electron Bands of Fullerites, ACS Nano, 2009, 3, 853-864; (b) M. Feng, J. Zhao, T. Huang, X. Zhu and H. Petek, The Electronic Properties of Superatom States of Hollow Molecules, Acc. Chem. Res., 2011, 44, 360-368; (c) L. Zoppi, L. Martin-Samos and K. K. Baldridge, Structure-Property Relationships of Curved Aromatic Materials from First Principles, Acc. Chem. Res., 2014, 47, 3310-3320.

12 T. J. Seiders, K. K. Baldridge, G. H. Grube and J. S. Siegel, Structure/Energy Correlation of Bowl Depth and Inversion Barrier in Corannulene Derivatives: Combined Experimental, and Quantum Mechanical Analysis, J. Am. Chem. Soc., 2001, 123, 517-525.
13 (a) L. Merz, M. Parschau, L. Zoppi, K. K. Baldridge, J. S. Siegel and K. H. Ernst, Reversible phase transitions in a buckybowl monolayer, Angew. Chem., Int. Ed., 2009, 48, 1966-1969; (b) T. Bauert, L. Zoppi, G. Koller, J. S. Siegel, K. K. Baldridge and K.-H. Ernst, Quadruple Anionic Buckybowls by Solid-State Chemistry of Corannulene and Cesium, J. Am. Chem. Soc., 2013, 135, 12857-12860.

14 V. M. Silkin, J. Zhao, F. Guinea, E. V. Chulkov, P. M. Echenique and $\mathrm{H}$. Petek, Image potential states in graphene, Phys. Rev. B: Condens. Matter Mater. Phys., 2009, 80, 121408.

15 (a) P. Hohenberg and W. Kohn, Inhomogeneous Electron Gas, Phys. Rev., 1964, 136, B864-B871; (b) W. Kohn and L. Sham, Self-Consistent Equations Including Exchange and Correlation Effects, Phys. Rev., 1965, 140, A1133-A1138.

16 A. J. Cohen, P. Mori-Sánchez and W. Yang, Challenges for Density Functional Theory, Chem. Rev., 2012, 112, 289-320.

17 G. Onida, L. Reining and A. Rubio, Electronic excitations: density-functional versus many-body Green's-function approaches, Rev. Mod. Phys., 2002, 74, 601-659.

18 (a) L. Hedin, New Method for Calculating the One-Particle Green's Function with Application to the Electron-Gas Problem, Phys. Rev., 1965, 139, A796-A823; (b) L. Hedin and S. Lundqvist, Effects of Electron-Electron and ElectronPhonon Interactions on the One-electron States in Solids, in Solid State Physics: Advances in research and Applications, ed. H. Ehrenreich, F. Seitz and D. Turnbull, Academic, New York, 1969, vol. 23.

19 N. Marom, F. Caruso, X. Ren, O. T. Hofmann, T. Korzdorfer, J. R. Chelikowsky, A. Rubio, M. Scheffler and P. Rinke, Benchmark of GW methods for azabenzenes, Phys. Rev. B: Condens. Matter Mater. Phys., 2012, 86, 245127.

20 T. Körzdörfer and N. Marom, Strategy for finding a reliable starting point for $G_{0} W_{0}$ demonstrated for molecules, Phys. Rev. B: Condens. Matter Mater. Phys., 2012, 86, 041110.

21 (a) J. B. Neaton, M. S. Hybertsen and S. G. Louie, Renormalization of Molecular Electronic Levels at Metal-Molecule Interfaces, Phys. Rev. Lett., 2006, 97, 216405; (b) J. M. GarciaLastra, C. Rostgaard, A. Rubio and K. S. Thygesen, Polarization-induced renormalization of molecular levels at metallic and semiconducting surfaces, Phys. Rev. B: Condens. Matter Mater. Phys., 2009, 80, 245427.

22 K. Kaasbjerg and K. S. Thygesen, Benchmarking $G W$ against exact diagonalization for semiempirical models, Phys. Rev. B: Condens. Matter Mater. Phys., 2010, 81, 085102.

23 (a) X. Blase, C. Attaccalite and V. Olevano, First-principles $G W$ calculations for fullerenes, porphyrins, phtalocyanine, and other molecules of interest for organic photovoltaic applications, Phys. Rev. B: Condens. Matter Mater. Phys., 2011, 83, 115103; (b) T. Körzdörfer, R. M. Parrish, N. Marom, J. S. Sears, C. D. Sherrill and J.-L. Brédas, Assessment of the performance of tuned range-separated hybrid density functionals in predicting accurate quasiparticle spectra, Phys. Rev. B: Condens. Matter Mater. Phys., 2012, 86, 205110; (c) F. Bruneval and M. A. L. Marques, Benchmarking the Starting Points of the $G W$ Approximation for Molecules, J. Chem. Theory Comput., 2013, 9, 324-329. 
24 M. Nooijen and R. J. Bartlett, Equation of motion coupled cluster method for electron attachment, J. Chem. Phys, 1995, 102, 3629-3646.

25 S. J. Grimme, Semiempirical GGA-type density functional constructed with a long-range dispersion correction, J. Comput. Chem., 2006, 27, 1787-1799.

26 M. W. Schmidt, K. K. Baldridge, J. A. Boatz, S. T. Elbert, M. S. Gordon, J. H. Jensen, S. Koseki, M. Matsunaga, K. A. Nguyen, S. Su, T. L. Windus and S. T. Elbert, General atomic and molecular electronic structure system, J. Comput. Chem., 1993, 14, 1347-1363.

27 P. Giannozzi, S. Baroni, N. Bonini, M. Calandra, R. Car, C. Cavazzoni, D. Ceresoli, G. L. Chiarotti, M. Cococcioni, I. Dabo, A. D. Corso, S. D. Gironcoli, S. Fabris, G. Fratesi, R. Gebauer, U. Gerstmann, C. Gougoussis, A. Kokalj, M. Lazzeri, L. Martin-Samos, N. Marzari, F. Mauri, R. Mazzarello, S. Paolini, A. Pasquarello, L. Paulatto, C. Sbraccia, S. Scandolo, G. Sclauzero, A. P. Seitsonen, A. Smogunov, P. Umari and R. M. Wentzcovitch, QUANTUM ESPRESSO: a modular and open-source software project for quantum simulations of materials, J. Phys.: Condens. Matter, 2009, 21, 395502.

28 L. Martin-Samos and G. Bussi, SaX: an open source package for electronic-structure and optical-properties calculations in the $G W$ approximation, Comput. Phys. Commun., 2009, 180, 1416-1425.

29 J. P. Perdew and A. Zunger, Self-interaction correction to density functional approximations for many-electron systems, Phys. Rev. B: Condens. Matter Mater. Phys., 1981, 23, 5048-5079.

30 M. Ernzerhof and G. E. Scuseria, Assessment of the PerdewBurke-Ernzerhof exchange-correlation functional, J. Chem. Phys., 1999, 110, 5029-5035.

31 M. A. Petrukhina and L. T. Scott, Fragments of Fullerenes and Carbon Nanotubes: Designed Synthesis, Unusual Reactions, and Coordination Chemistry, Wiley, 2011.

32 V. K. Voora, L. S. Cederbaum and K. D. Jordan, Existence of a Correlation Bound s-Type Anion State of C60, J. Phys. Chem. Lett., 2013, 4, 849-853.

33 (a) T. Huang, J. Zhao, M. Feng, H. Petek, S. Yang and L. Dunsch, Superatom orbitals of Sc3N@C80 and their intermolecular hybridization on $\mathrm{Cu}(110)-(2 \times 1)-\mathrm{O}$ surface, Phys. Rev. B: Condens. Matter Mater. Phys., 2010, 81, 085434; (b) M. Feng, Y. Shi, C. I. Lin, J. Zhao, F. Liu, S. Yang and H. Petek, Energy stabilization of the s-symmetry superatom molecular orbital by endohedral doping of C82 fullerene with a lanthanum atom, Phys. Rev. B: Condens. Matter Mater. Phys., 2013, 88, 075417. 